\title{
Diversité Et Structure Floristiques Sous Des Peuplements d'Acacias Australiens En Zone Forestière De La Côte d'Ivoire
}

\author{
Kouadio Kouassi Richard, MA \\ Bakayoko Adama, PhD
}

UFR des Sciences de la Nature, Université Nangui Abrogoua, Côte d'Ivoire N'guessan Kanga Anatole, PhD

Centre National de Recherche Agronomique (CNRA), Côte d'Ivoire Konan Djezou, PhD

UFR des Sciences de la Nature, Université Nangui Abrogoua, Côte d'Ivoire doi: 10.19044/esj.2016.v12n35p229 URL:http://dx.doi.org/10.19044/esj.2016.v12n35p229

\begin{abstract}
Since thirty years, four Australian Acacias species are introduced and studied in Anguédédou Research station. The station research is located in evergreen forest in the south of Côte d'Ivoire. The objective of the introduction of these species, is to rehabilitate degraded grounds and fallow after growing. The study aims at appreciating the impact of Acacia species on floristic diversity and the regeneration of forest species. For collecting data, we use quadrat method ( $35 \times 50 \mathrm{~m}$ and $6 \times 6 \mathrm{~m}$ ). This method permitted the inventory of 212 species on 1.05 ha. Among these species, $1.89 \%$ are Ivorian endemic species and $11.32 \%$ are endemic of West African flora. The flora under the different species of Acacia are relatively diversified and dense. The majority of plant inventoried under Acacia species have small diameters.

The highest specific richness average per plots $\left(36 \mathrm{~m}^{2}\right)$ were noted in the 11 years old parcels of Acacia mangium (26.20 \pm 2.34$)$ and 27 years old parcels

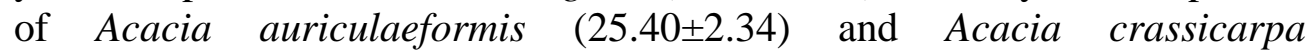
$(30.60 \pm 2.34)$. The mean values of diameters $(\mathrm{dbh} \geq 2.5 \mathrm{~cm})$ fluctuate from $2.94 \pm 0.56 \mathrm{~cm}$ for the 8 years old stands of Acacia mangium to $8.09 \pm 0.56 \mathrm{~cm}$ for the 27 years old settlements of Acacia crassicarpa. The results show that leguminous trees can be used for recolonization of the deforested areas in order to rebuild quickly their plant biodiversity.
\end{abstract}

Keywords: Acacia, biodiversity, evergreen forest, Ivory Coast, Anguédédou 


\section{Résumé}

Depuis une trentaine d'années, quatre espèces d'Acacias australiens sont introduites et étudiées en zone de forêt dense humide sempervirente de Côte d'Ivoire, dans la Station de Recherche Forestière d'Anguédédou. L'objectif de l'introduction de ces espèces, est la réhabilitation des sols dégradés, abandonnés en jachères après des pratiques culturales. L'étude vise à apprécier leur impact sur la diversité floristique sous les peuplements de trois de ces espèces d'Acacia. Des relevés de surface (35 x $50 \mathrm{~m}$ et 6 x $6 \mathrm{~m}$ ) réalisés dans chacun de ces peuplements ont permis de recenser 212 espèces végétales sur 1,05 ha de parcelles inventoriées, soit une moyenne de 201,9 espèces/ha. Parmi ces espèces, 1,89 \% figurent sur la liste des espèces endémiques ivoiriennes et $11,32 \%$ sont endémiques à la flore ouestafricaine. Les flores sous les différents peuplements étudiés sont relativement diversifiées et denses, avec majoritairement des individus de petits diamètres. Les richesses spécifiques moyennes par placettes $\left(36 \mathrm{~m}^{2}\right)$ les plus élevées ont été enregistrées dans la parcelle de 11 ans d'Acacia mangium $(26,20 \pm 2,34)$ et celles âgées de 27 ans d'Acacia auriculaeformis $(25,40 \pm 2,34)$ et d'Acacia crassicarpa $(30,60 \pm 2,34)$. Les valeurs moyennes de diamètres (d.b.h $\geq 2,5 \mathrm{~cm}$ ) varient de $2,94 \pm 0,56 \mathrm{~cm}$ pour le peuplement de 8 ans d'Acacia mangium à 8,09 $\pm 0,56 \mathrm{~cm}$ pour le peuplement d'Acacia crassicarpa âgé de 27 ans. Les résultats montrent que les Légumineuses arborescentes peuvent servir à recoloniser les surfaces déforestées en vue de reconstituer rapidement leur biodiversité végétale.

Mot clés: Acacia, biodiversité, forêt dense humide sempervirente, Côte d’Ivoire, Anguédédou

\section{Introduction}

La reconstitution des couverts forestiers est l'un des nombreux défis auxquels s'attèlent les politiques de gestion durable des ressources naturelles. Chaque année, en moyenne 21 millions d'hectares de forêts tropicales à l'échelle planétaire sont convertis de manière permanente en surface agricoles et en infrastructures, ce qui représente environ 1,2 \% de forêts en milieu tropical coupées ou détruites. Ce rythme effréné de déforestation et de dégradation des forêts constitue une menace pour l'environnement en termes de survie de nombreuses espèces végétales et animales. Depuis quelques décennies, la forêt dense humide de la Côte d'Ivoire fait place à des portions de forêts en dégradation ou en reconstitution (N'Guessan et al., 2006). À l'indépendance du pays en 1960, la stratégie économique du gouvernement était d'axer le développement du pays sur l'agriculture industrielle. Cette option basée sur le binôme café-cacao et les autres cultures de rente (palmier 
à huile, hévéa, ananas, etc.) a occasionné une perte massive des écosystèmes forestiers (Bertrand, 1983). Estimée à 16 millions d'hectares à la fin du 19ème siècle, la couverture forestière a drastiquement régressé, au point d’être évaluée à environ 1,385 millions ha en 2000 (Koné et al., 2014).

La forte pression anthropique sur les ressources forestières de la Côte d'Ivoire conduisant à la réduction de leurs surfaces et à leur fragmentation constitue une menace majeure pour la biodiversité (Quinn and Harrison, 1988; Gibbs, 1998). Outre la réduction de la biodiversité ces actions, l'empêchent également de se reconstituer. Ainsi, on assiste à la disparition et la raréfaction d'un nombre important d'espèces végétales que contiennent ces écosystèmes. Les préoccupations que suscite la destruction des forêts ivoiriennes se sont considérablement amplifiées au cours des deux dernières décennies, et ont appelé diverses initiatives à court et moyen termes. Ces initiatives visent à reconstituer le couvert forestier national et à élaborer des stratégies et des actions en faveur de l'aménagement forestier durable. Parmi elles, l'introduction et l'étude des Légumineuses arborescentes australiennes, à croissance rapide telles que les Acacias à Port- Bouët et à Assinie-France en zone littorale, à Anguédédou, Dabou, La Mé et Sangoué (N’Guessan, 1991 ; Allou et al., 2001 ; N’Goran et al., 2003). Les objectifs assignés à ces essais sont de divers ordres : arrêter la destruction abusive des massifs forestiers, restaurer les forêts dégradées, restituer et maintenir la fertilité des sols et assurer un approvisionnement soutenu en bois d'œuvre et de service (N'Guessan, 2006). Par ailleurs, les solutions envisageables doivent tenir aussi compte d'une approche globale dans un contexte villageois et périurbain.

En Côte d’Ivoire, plusieurs travaux ont été réalisés pour comprendre l'influence des Légumineuses arborescentes sur les productions végétales. Elles réduisent l'enherbement qui est un facteur limitant la production agricole (Djaha et al., 2009). L’association Légumineuse arborescentecocotier a donné des résultats intéressants. Ainsi, Dupuy et N’Guessan (1991) et Zakra (1996) ont montré que cette méthode agroforestière est une alternative pour la régénération des cocoteraies. L’étude s’intéresse à trois des quatre espèces d'acacias australiens qui ont été introduits à partir de 1982 dans la forêt classée de l'Anguédédou dans l’objectif de réhabiliter les sols abandonnés en jachères après des pratiques culturales.

Outre les précédents essais effectués à des fins agricoles et sylvicoles, la présente approche veut utiliser les avantages qu’offrent les Légumineuses arborescentes pour stimuler la régénération forestière. Il s’agit notamment de : (i) la présence d'ombre qui est propice à l'installation des jeunes pousses, (ii) l'amélioration de la qualité du sol, favorable à l'établissement de la surface forestière et (iii) la réduction de l'enherbement qui permet la bonne installation des espèces forestières surtout ligneuses. Cette étude se propose 
d'être une alternative de reboisement et d'amélioration de la biodiversité. Spécifiquement, elle cherche à évaluer la diversité des espèces végétales et la structure de la flore sous différents peuplements de ces espèces végétales introduites en zone de forêt dense humide sempervirente de la Côte d'Ivoire.

\section{Matériels et méthodes d'étude Site d'étude}

La Forêt Classée de l’Anguédédou, est située à la périphérie, à 20 km au nord de la capitale $\left(5^{\circ} 22^{\prime}-5^{\circ} 26^{\prime} \mathrm{N}\right.$ et $\left.4^{\circ} 04^{\prime}-4^{\circ} 13^{\prime} \mathrm{W}\right)$ dans le Sud forestier de la Cote d'Ivoire (Figure 1). C'est en son sein que se trouve la Station de recherche forestière CNRA d'Anguédédou qui est le cadre de réalisation de cette étude. Cette station couvre une superficie de 176 ha et comporte de nombreux peuplements monospécifiques et en mélange de diverses essences forestières réalisées sur d'anciennes jachères agricoles (Adou, 1986). La station jouit d'un climat tropical chaud humide, de type subéquatorial. Ce régime climatique est caractérisé par une température moyenne annuelle de $26^{\circ} \mathrm{C}$ à $27^{\circ} \mathrm{C}$, et un fort taux d'humidité (de 80 à $90 \%$ ). les précipitations sont abondantes et se répartissent sur quatre saisons, à savoir deux saisons pluvieuses et deux saisons sèches (Eldin, 1971). La pluviométrie annuelle moyenne couvrant la période de 1999 à 2014 est de 2004,29 mm.

La végétation de la station appartient au secteur ombrophile du domaine Guinéen. Le climax dominant est la forme sempervirente de la forêt dense humide et la défeuillaison n'affecte jamais l'ensemble de la végétation (Guillaumet et Adjanohoun, 1971). C'est une forêt psammophile caractérisée par l'association Turraeanthus africanus Pellegr. (Meliaceae) - Heisteria parvifolia Smith. (Olacaceae). Les sols du site d'étude qui reposent sur des sables tertiaires à plusieurs faciès, sont du type ferralitique fortement désaturé, appauvri en argile. Ce sont des sols très profonds, légers, sabloargileux et ferrugineux (N’Guessan, 2006). 


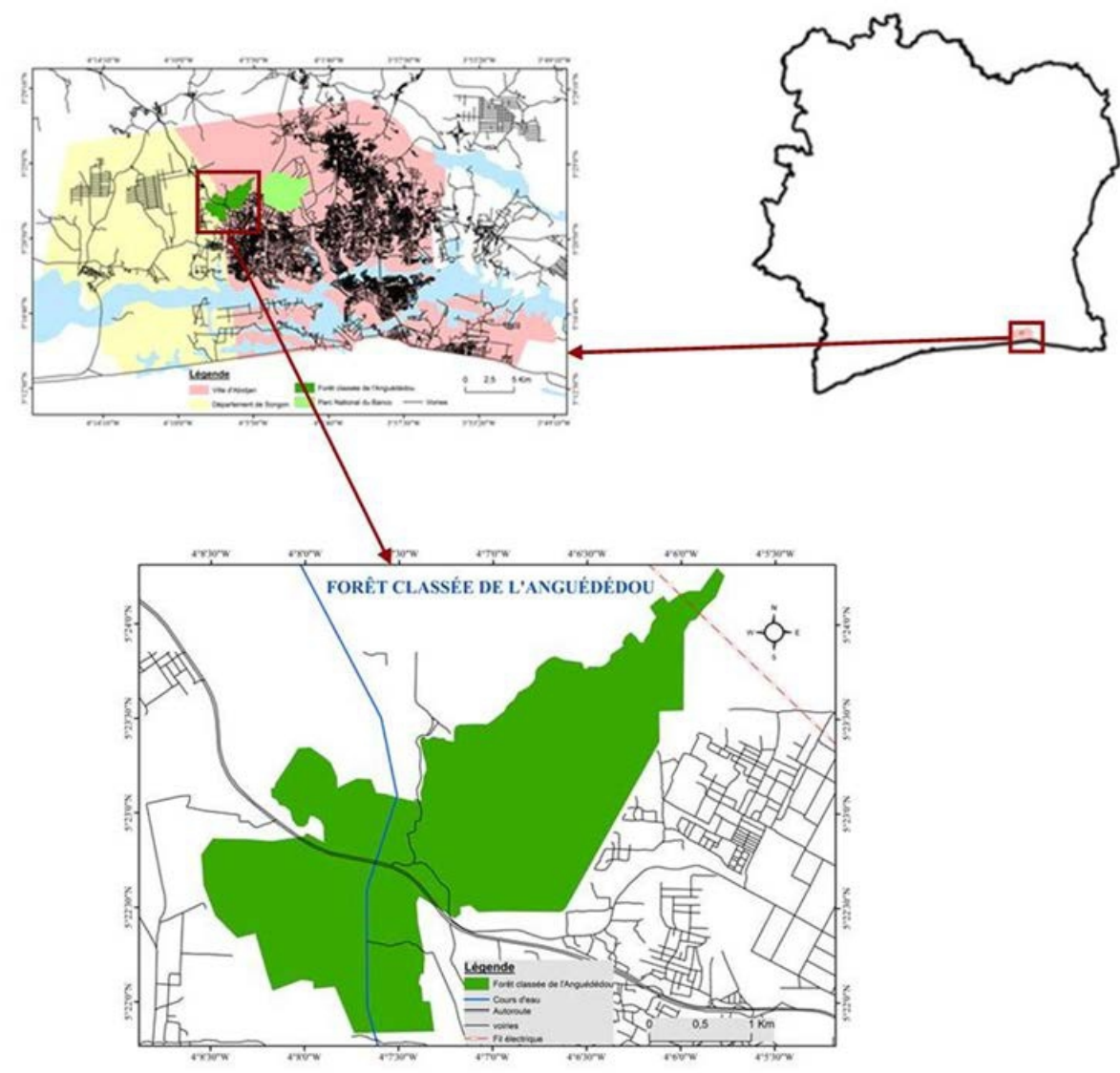

Figure 1 : localisation de la zone d'étude

\section{Matériels d'étude}

\section{Matériel technique}

Il se compose de fiches de relevés pour inscrire les noms des espèces inventoriées et de rubans gradués les mesures de circonférences des individus. Nous avons eu besoin de papiers journaux pour conserver les échantillons récoltés et d'un four pour le séchage de ces récoltes. Nous avons aussi manipulé un GPS (Global System Position) pour mentionner les coordonnées géographiques.

Matériel biologique

Il est constitué des trois espèces d'Acacias australiens retenues (Tableau I) et de toutes les espèces végétales inventoriées. 
Tableau I : Description des trois espèces d'Acacia retenues

\begin{tabular}{|c|c|c|c|}
\hline Espèce & Ecologie & Description botanique & Usages \\
\hline $\begin{array}{l}\text { Acacia mangium Willd } \\
\text { (Fabaceae) }\end{array}$ & $\begin{array}{c}\text { Origine : Indonésie, } \\
\text { Papouasie-Nouvelle- } \\
\text { Guinée, et Australie. } \\
\text { Altitude : } 0-780 \mathrm{~m} \\
\text { Pluies : } 1000- \\
\text { 4500mm } \\
\text { Température : } 15-35^{\circ} \\
\text { C }\end{array}$ & $\begin{array}{c}\text { Hauteur : } 30 \text { m Feuilles : } \\
\text { phyllodes (11 à } 27 \mathrm{~cm} \\
\text { de long sur } 3 \text { à } 10 \mathrm{~cm} \text { de } \\
\text { large) } \\
\text { Fruits : gousses se } \\
\text { recroquevillant en } \\
\text { spirale à maturité. }\end{array}$ & $\begin{array}{l}\text { Produits alimentaires, } \\
\text { fourrages, bois- } \\
\text { énergie, bois d'œuvre, } \\
\text { tanins, reboisement, } \\
\text { conservation et } \\
\text { amélioration du sol, } \\
\text { agroforesterie, } \\
\text { aménagement urbain. }\end{array}$ \\
\hline $\begin{array}{l}\text { Acacia auriculaeformis } \\
\text { Benth (Fabaceae) }\end{array}$ & $\begin{array}{c}\text { Origine : Indonésie, } \\
\text { Papouasie-Nouvelle- } \\
\text { Guinée, et Australie. } \\
\text { Altitude : 0-1000 m } \\
\text { Pluies : 600-3500mm } \\
\text { Température : } 15-35^{\circ} \\
\text { C }\end{array}$ & $\begin{array}{l}\text { Hauteur : } 30 \mathrm{~m} \text { Feuilles : } \\
\text { (10 à } 16 \mathrm{~cm} \text { de long et } \\
1,5 \text { à } 2,5 \mathrm{~cm} \text { de large) } \\
\text { Fruits : gousses ondulées } \\
\text { et irrégulièrement } \\
\text { spiralées. }\end{array}$ & $\begin{array}{c}\text { Produits alimentaires, } \\
\text { apiculture, fourrages, } \\
\text { bois-énergie, bois } \\
\text { d'œuvre, tanins, } \\
\text { conservation et } \\
\text { amélioration du sol, } \\
\text { agroforesterie, } \\
\text { aménagement urbain. }\end{array}$ \\
\hline $\begin{array}{l}\text { Acacia crassicarpa } \\
\text { Cunn. ex Benth } \\
\text { (Fabaceae) }\end{array}$ & $\begin{array}{c}\text { Origine : Indonésie, } \\
\text { Papouasie-Nouvelle- } \\
\text { Guinée, et Australie. } \\
\text { Altitude : } 0-450 \mathrm{~m} \\
\text { Pluies : 500-3500 mm } \\
\text { Température : } 15-34^{\circ} \\
\text { C }\end{array}$ & $\begin{array}{c}\text { Hauteur : } 30 \mathrm{~m} \text { Feuilles : } \\
\text { phyllodes falciformes ( } 8 \\
\text { à } 27 \mathrm{~cm} \text { de long sur } 1 \text { à } \\
4,5 \mathrm{~cm} \text { de large) Fruits : } \\
\text { gousses ligneuses plates, } \\
\text { ovoïdes-oblongues et } \\
\text { transversalement } \\
\text { veinées. }\end{array}$ & $\begin{array}{l}\text { Bois-énergie, bois } \\
\text { d’œuvre, tanins, } \\
\text { conservation et } \\
\text { amélioration du sol. }\end{array}$ \\
\hline
\end{tabular}

\section{Méthodes d'étude}

\section{Dispositif de collecte des données}

Le choix des essais à étudier a tenu compte du type d'espèce d'Acacia et de l'âge des peuplements des espèces considérées. Trois espèces ont été retenues et le nombre de peuplements de chaque espèce choisie varie en fonction des parcelles disponibles. Le dispositif expérimental comprend trois types de peuplements: le peuplement très jeune d'Acacia mangium âgé de 3 ans (PMan3), les peuplements jeunes, âgés de 8 ans d'Acacia mangium (PMan8) et d'Acacia auriculaeformis (PAur8) et le dernier type, constitué par les peuplements plus ou moins adultes d'Acacia mangium de 11 ans (PMan11), Acacia auriculaeformis de 27 ans (PAur27) et Acacia crassicarpa de 27 ans (PCra27).

\section{Etude floristique}

\section{Richesse et diversité floristiques}

La richesse floristique est le nombre d'espèces recensées dans un milieu, sans tenir compte de leur fréquence, leur abondance, ni même de leur taille ou leur productivité (Kouamé, 1998). Elle a été estimée par la méthode de relevés de surface pour l'inventaire de toutes les espèces végétales rencontrées. Dans chaque peuplement, la collecte des données a été effectuée 
dans des placettes rectangulaires de 50 x 35 m et des placettes carrées de 6 x $6 \mathrm{~m}$. La nomenclature adoptée pour la flore est la classification APG III (2009).

La diversité floristique recouvre la richesse floristique et l'équitabilité qui est la manière dont les individus des espèces rencontrées se répartissent. L’étude l'a abordée au moyen des indices de Shannon et de Piélou exprimés par les équations suivantes:

$$
\mathbf{H}=-\sum(\mathbf{n i} / \mathbf{N}) \log _{2}(\mathbf{n i} / \mathbf{N}) \text { et } \mathbf{E}=\mathbf{H} / \ln \mathrm{S}
$$

H étant l'indice de Shannon ; ni, le nombre d'individus d'une espèce $\mathrm{i}$; $\mathrm{N}$, le nombre total d'individus de toutes les espèces; $\mathrm{E}$, l'indice d'équitabilité de Piélou ; et $\mathrm{S}$, le nombre total d'espèces d'un biotope.

\section{Types morphologiques}

Les types morphologiques des espèces ont été précisés en référence à Aké-Assi (2001-2002). Il s’agit des espèces arborescentes, des espèces lianescentes et des espèces herbacées.

\section{Etude structurale}

\section{Classes de diamètres}

Pour l'évaluation des paramètres structuraux, tous les individus des espèces arborescentes ont été pris en compte et mesurés. Dans chacune des placettes carrées, ces inventaires dendrométriques ont concerné les individus de dbh supérieur à $2,5 \mathrm{~cm}$. Ce choix opéré nous a permis de prendre en compte une grande partie des individus de sous-bois. Tous les diamètres enregistrés ont été répartis en quatre classes de diamètres : $[2,5 \mathrm{~cm} ; 5 \mathrm{~cm}[$; [5 cm;10 cm[ ; $[10 \mathrm{~cm} ; 15 \mathrm{~cm}[;[15 \mathrm{~cm} ; \infty[$.

\section{Densité moyenne de tiges (D) et surface terrière (St)}

La densité moyenne permet d'évaluer la densité des individus juvéniles et celle des individus adultes dans chacun des biotopes. Elle est déterminée par le nombre de tiges à l'hectare. La surface terrière moyenne renseigne sur la dominance relative de chaque espèce dans le peuplement d'un biotope donné. Elle est déterminée pour évaluer la surface occupée par les sections des fûts à 1,30 m du sol, dans différents biotopes. Elle s'exprime en $\mathrm{m}^{2} /$ ha. La surface terrière d'un arbre est d'autant plus élevée que l'arbre est gros. Celle d'un peuplement est d'autant plus élevée que celui-ci est dense. Ces paramètres sont calculés selon les équations suivantes :

$$
\mathbf{D}=\mathbf{n} / \mathbf{S} \text { et } \mathbf{S t}=\mathbf{C}^{\mathbf{2}} / \mathbf{4} \boldsymbol{\pi}
$$

D étant la densité des tiges; n, le nombre total d'individus inventoriés dans le biotope ; S, l'aire totale échantillonnée dans le milieu ; St la surface terrière et $\mathrm{C}$ la circonférence. 


\section{Etude statistique des données}

Toutes les données recueillies ont été saisies et dépouillées sur Excel 2013 du système Windows 8. Le logiciel PAST a servi à calculer les indices de diversité (Shannon) et d’équitabilité (Piélou). L’identification des différents biotopes a nécessité une analyse de variance à un facteur et la comparaison des moyennes (ANOVA 1) des paramètres floristiques (richesse, composition, indices de diversité) et structuraux (densité et aire basale) calculés. Le niveau de significativité choisi pour ces analyses, est de $5 \%$ ( $p$-value $\leq 0,05)$. Lorsque la différence est significative, le test de Tukey a été réalisé pour classer et distinguer lesquels des groupes sont différents. Le logiciel XLSTAT a été utilisé pour la réalisation de ces tests.

\section{Résultats}

\section{Etude floristique}

\section{Richesse et diversité floristiques}

Deux cent douze (212) espèces reparties en 158 genres classés dans 67 familles ont été inventoriées dans l'ensemble des peuplements. Les espèces les plus rencontrées sont Angylocalyx oligophyllus (Baker) Baker f., Baphia nitida Lodd., Dichapetalum pallidum (Oliv.) Engl., Epinetrum cordifolium Mangenot \& Miege, Icacina mannii Oliv., Microdesmis keayana J.Léonard, Salacia nitida (Benth.) N.E.Br.. Les familles les mieux représentées en nombre d'espèces (Tableau II) sont celles des Fabaceae, Apocynaceae, Rubiaceae, Annonaceae, Euphorbiaceae, Lamiaceae, Mennispermaceae et Sapindaceae. Dans l'ensemble des relevés, elles representent 39,62 \% des espèces de la flore inventoriée. La famille des Fabaceae est prépondérante dans tous les biotopes, sauf dans PAur27 et PCra27 où elle est respectivement codominante avec les Apocynaceae et les Annonaceae.

Tableau II : Proportions d'espèces des familles botaniques les mieux représentées dans les différents peuplements d'Acacia

\begin{tabular}{cccccccc}
\multirow{n}{*}{$\begin{array}{c}\text { Familles } \\
\text { botaniques }\end{array}$} & PAur8 & PAur27 & PCra27 & PMan3 & PMan8 & PMan11 & Ens.rel* $^{*}$ \\
\cline { 2 - 7 }$y$ & 16,18 & 10,53 & 7,50 & 10,71 & 15,38 & 8,93 & 8,49 \\
Fabaceae & 7,35 & 10,53 & 5,83 & 7,14 & 10,77 & 8,93 & 7,08 \\
Apocynaceae & 2,94 & 3,95 & 3,33 & 3,57 & 3,08 & 3,57 & 5,66 \\
Rubiaceae & 4,41 & 2,63 & 7,50 & 1,79 & 4,62 & 3,57 & 4,72 \\
Annonaceae & 2,94 & 1,32 & 4,17 & 0,00 & 0,00 & 4,46 & 3,77 \\
Euphorbiaceae & 2,94 & 3,95 & 1,67 & 3,57 & 4,62 & 3,57 & 3,30 \\
Lamiaceae & 2,94 & 2,63 & 4,17 & 3,57 & 4,62 & 4,46 & 3,30 \\
Mennispermaceae & 5,88 & 5,26 & 4,17 & 5,36 & 4,62 & 4,46 & 3,30 \\
Sapindaceae & 45,58 & 40,80 & 38,34 & 35,71 & 47,71 & 41,95 & 39,62 \\
\hline Total & \multicolumn{7}{c}{ Ens.rel : ensemble des relevés }
\end{tabular}

Les richesses floristiques moyennes dans les placettes varient de 15,00 espèces pour PMan3 à 30,60 espèces pour PCra27 (Tableau III). Les 
différences observées entre les valeurs moyennes sont significatives $(F=$ 4,794; $P=0,004$ ) et trois catégories de biotopes se dégagent. La première catégorie qui est celle des peuplements dont la richesse floristique est la plus élevée, est composée des peuplements adultes. Il s’agit de PAur 27 $(25,40 \pm 2,34)$, PCra $27(30,60 \pm 2,34)$ et PMan11 $(26,20 \pm 2,34)$. Dans le deuxième groupe, on retrouve les peuplements dits jeunes. Cette catégorie est caractérisée par des richesses floristiques moyennes $(24,20 \pm 2,34$ pour

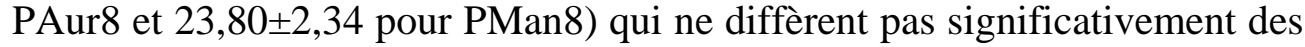
deux autres groupes. Le troisième groupe est représenté par les peuplements de plus faible richesse spécifique. On y retrouve le peuplement dit très jeune,

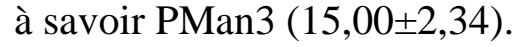

Des différences significatives $(F=3,812 ; P=0,011)$ sont observées au niveau des valeurs moyennes de l'indice de Shannon qui exprime la diversité (Tableau III). Trois groupes de biotopes se distinguent. Le peuplement PCra27 dont l'indice de Shannon $(3,02 \pm 0,12)$ est le plus élevé est le représentant du premier groupe. Le deuxième groupe est formé par les peuplements dont les valeurs moyennes d'indices de Shannon sont intermédiaires à celles du premier groupe et du troisième groupe. Ce groupe renferme PAur8 (2,82 $\pm 0,12)$, PMan8 $(2,83 \pm 0,12)$, PMan11 $(2,66 \pm 0,12)$ et

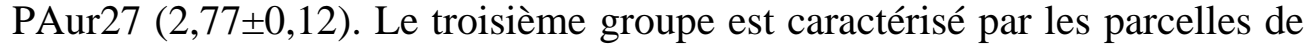
plus faible valeur d'indice de Shannon. Cette dernière section est constituée

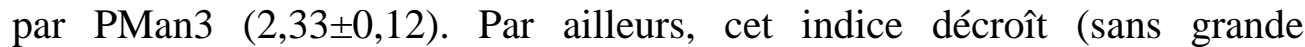
différence) d'un stade jeune à un stade adulte. Aucune différence significative $(F=1,118 ; P=0,377)$ n’est observée pour les valeurs moyennes d’indice d'équitabilité de Piélou qui sont très élevées (Tableau III).

Tableau III : Richesse et diversité floristiques des différents biotopes

\begin{tabular}{ccccc}
\cline { 2 - 5 } Biotopes & $\begin{array}{c}\text { Nombre } \\
\text { espèces/biotope }\end{array}$ & $\begin{array}{c}\text { Nombre moyen } \\
\text { espèces /placette }\end{array}$ & $\begin{array}{c}\text { Diversité de } \\
\text { Shannon (H') }\end{array}$ & $\begin{array}{c}\text { Equitabilité } \\
\text { de Piélou (J') }\end{array}$ \\
\hline PAur8 & 69 & $24,20 \pm 2,34 \mathrm{~b}$ & $2,82 \pm 0,12 \mathrm{ab}$ & $0,92 \pm 0,01 \mathrm{a}$ \\
PAur27 & 76 & $\mathbf{2 5 , 4 0 \pm 2 , 3 4 a}$ & $2,77 \pm 0,12 \mathrm{ab}$ & $0,91 \pm 0,01 \mathrm{a}$ \\
PCra27 & 120 & $\mathbf{3 0 , 6 0} \pm \mathbf{2 , 3 4 a}$ & $\mathbf{3 , 0 2} \pm \mathbf{0 , 1 2 a}$ & $0,93 \pm 0,01 \mathrm{a}$ \\
PMan3 & 56 & $15,00 \pm 2,34 \mathrm{~b}$ & $2,33 \pm 0,12 \mathrm{~b}$ & $0,91 \pm 0,01 \mathrm{a}$ \\
PMan8 & 65 & $23,80 \pm 2,34 \mathrm{~b}$ & $2,83 \pm 0,12 \mathrm{ab}$ & $0,95 \pm 0,01 \mathrm{a}$ \\
PMan11 & 112 & $\mathbf{2 6 , 2 0 \pm \mathbf { 2 , 3 4 a }}$ & $2,66 \pm 0,12 \mathrm{ab}$ & $0,91 \pm 0,01 \mathrm{a}$ \\
\hline Paramètres & & $F=4,794$ & $F=3,812$ & $F=1,118$ \\
statistiques & & $P=0,004$ & $P=0,011$ & $P=0,377$ \\
\hline
\end{tabular}

*Pour chaque colonne, les valeurs moyennes affectées de la même lettre ne sont pas significativement différentes au seuil de 5\% (test de Tukey) et les valeurs en gras sont les valeurs maximales.

\section{Types morphologiques}

Les proportions des différents types morphologiques varient d'une part au sein de chaque biotope, et d'autre part entre les différents biotopes (Tableau IV). 
Les proportions d'espèces arborescentes dans les différents peuplements d'Acacia varient de 37,04 \% (PMan11) à 68,74 \% (PCra27). Ces proportions présentent des différences significatives d'un biotope à un autre. Trois ensembles de peuplements ont été identifiés à l'aide du test de Tukey. Le premier ensemble est celui des peuplements à fort taux d'espèces

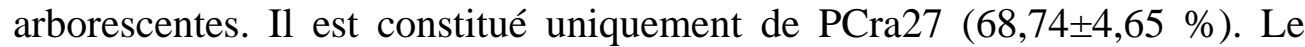
second groupe se singularise par des peuplements dont les pourcentages d'espèces arborescentes sont compris entre ceux du premier ensemble et de la troisième catégorie. Dans ce groupe, on retrouve les deux peuplements dits

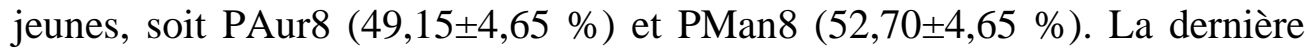
catégorie comprend des parcelles aux plus faibles proportions en espèces

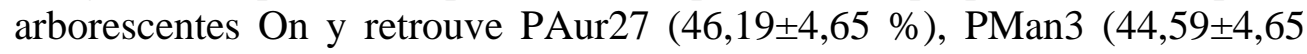
$\%)$ et PMan11 (37,04 $\pm 4,65 \%)$.

Concernant les proportions d'espèces lianescentes, les différences entre les peuplements sont significatives $(F=3,479 ; P=0,017)$. Trois groupes de biotopes se détachent et la tendance observée est inversée par rapport aux espèces arborescentes. Le premier groupe est marqué par les biotopes les

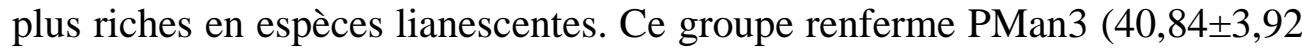

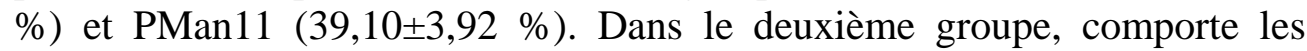
biotopes dont les proportions en espèces lianescentes sont intermédiaires aux

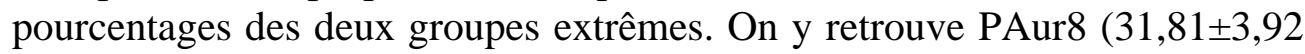

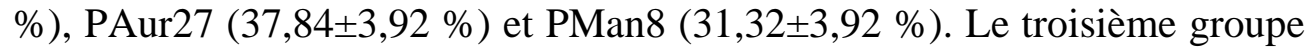
est celui des milieux les moins riches en lianes. Il est constitué uniquement

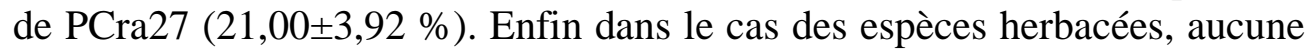
différence significative n’a été observée pour leurs proportions dans les différents peuplements étudiés $(F=1,359 ; P=0,274)$.

Tableau IV : Types morphologiques des différentes catégories de peuplements d'Acacia

\begin{tabular}{cccc} 
& \multicolumn{3}{c}{ Types morphologiques (\%) } \\
\cline { 2 - 4 } Biotopes & $\begin{array}{c}\text { Espèces } \\
\text { arborescentes }\end{array}$ & Espèces lianescentes & Espèces herbacées \\
& $49,15 \pm 4,65 \mathrm{ab}$ & $31,81 \pm 3,92 \mathrm{ab}$ & $19,05 \pm 3,91 \mathrm{a}$ \\
PAur8 & $46,19 \pm 4,65 \mathrm{~b}$ & $37,84 \pm 3,92 \mathrm{ab}$ & $15,97 \pm 3,91 \mathrm{a}$ \\
PAur27 & $\mathbf{6 8 , 7 4} \pm \mathbf{4 , 6 5 a}$ & $21,00 \pm 3,92 \mathrm{~b}$ & $10,27 \pm 3,91 \mathrm{a}$ \\
PCra27 & $44,59 \pm 4,65 \mathrm{~b}$ & $\mathbf{4 0 , 8 4} \pm 3, \mathbf{9 2 a}$ & $14,57 \pm 3,91 \mathrm{a}$ \\
PMan3 & $52,70 \pm 4,65 \mathrm{ab}$ & $31,32 \pm 3,92 \mathrm{ab}$ & $15,98 \pm 3,91 \mathrm{a}$ \\
PMan8 & $37,04 \pm 4,65 \mathrm{~b}$ & $\mathbf{3 9 , 1 0} \pm 3,92 \mathrm{a}$ & $23,86 \pm 3,91 \mathrm{a}$ \\
PMan11 & 49,00 & 29,72 & 21,23 \\
Total & $F=5,286$ & $F=3,479$ & $F=1,359$ \\
Paramètres & $P=0,002$ & $P=0,017$ & $P=0,274$ \\
statistiques & &
\end{tabular}

*Pour chaque colonne, les valeurs moyennes affectées de la même lettre ne sont pas significativement différentes au seuil de 5\% (test de Tukey) et les valeurs en gras sont les valeurs maximales. 


\section{Etude structurale}

\section{Classes de diamètres}

On observe une distribution des individus dans toutes les classes de diamètres au niveau de PCra27 (Figure 2). Dans la classe de 2,5 à $5 \mathrm{~cm}$ de diamètre, PMan11 affiche la plus forte densité (2666,67 tiges/ha). Il est suivi respectivement par PAur27 (2277,78 tiges/ha), PCra27 (1888,89 tiges/ha), PMan8 (1222,22 tiges/ha) et PAur8 (555,56 tiges/ha). Par ailleurs, aucun individu de plus de $2,5 \mathrm{~cm}$ de diamètre n'a été rencontré dans PMan3. Seuls PCra27 et PAur27 présentent des individus de diamètres supérieurs à $5 \mathrm{~cm}$. Dans la classe de 5 à $10 \mathrm{~cm}$ de diamètre, l'on note 1055,56 tiges/ha pour PCra27 et 222,22 tiges/ha pour PAur27. Au niveau de la classe de 10 à 15 cm de diamètre, on enregistre 333,33 tiges/ha pour PCra27 et 55,56 tiges/ha pour PAur27. Enfin, PCra27 est le seul biotope dont les individus sont distribués dans la classe de plus de $15 \mathrm{~cm}$ de diamètre avec 444,44 tiges/ha.

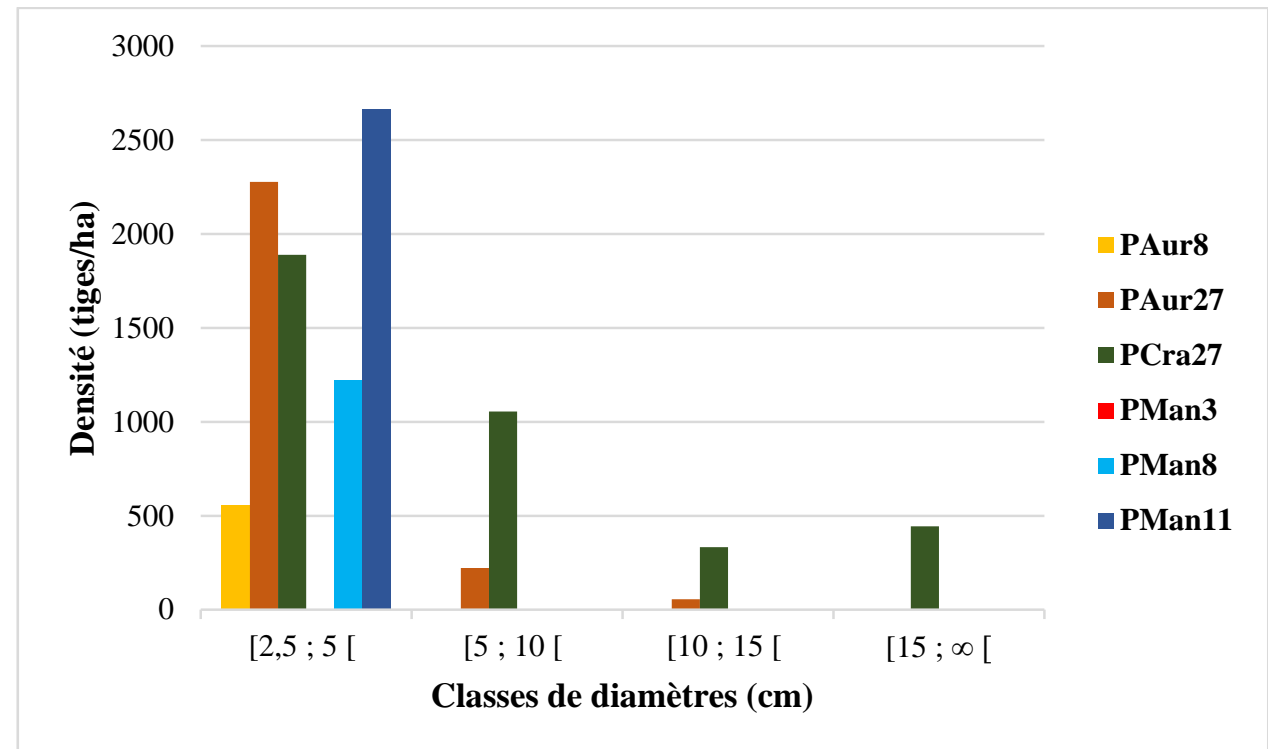

Figure 2 : distribution des individus dans les classes de diamètres dans les différents biotopes

L'étude du diamètre moyen des individus de chaque biotope révèle des valeurs maximales pour PCra27 $(8,09 \pm 0,56 \mathrm{~cm})$ et nulles pour PMan3 $(0,00 \pm 0,00 \mathrm{~cm})$ (Tableau V). On observe des différences statistiques $(P=21,946 ; F=0,0001)$ entre les peuplements, qui donnent trois groupes. Le premier groupe avec des valeurs élevées $(8,09 \pm 0,56 \mathrm{~cm})$ est composé uniquement de PCra27. Le second groupe renferme les peuplements ayant des valeurs moyennes faibles. On note les valeurs de 3,01 $\pm 0,56 \mathrm{~cm}$ pour PAur8, 3,57 $\pm 0,56 \mathrm{~cm}$ pour PAur27, 2,94 $\pm 0,56 \mathrm{~cm}$ pour PMan8 et 3,05 $\pm 0,56$ $\mathrm{cm}$ pour PMan11. Ce groupe est tout de même dominé par PAur27 


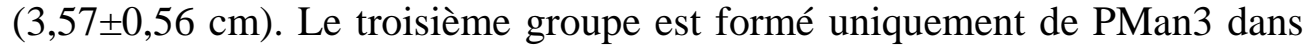
lequel aucun diamètre supérieur à 2,5 cm n’a été noté.

\section{Densité et surface terrière}

L’étude statistique de la densité moyenne des tiges à l'hectare pour les individus de diamètre supérieur à $2,5 \mathrm{~cm}$ montre des différences significatives $(P=20,146 ; F=0,0001)$. Cinq groupes de biotopes se dégagent (Tableau V). Dans le premier groupe, on retrouve les peuplements dont les valeurs de cette variable sont les plus élevées. Il s’agit de PMan11

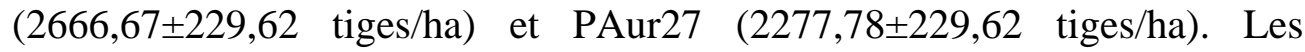
deuxième, troisième et quatrième groupes sont respectivement constitués de PCra27 (1888,89 $\pm 229,62$ tiges/ha) d'une part, PMan8 (1222,22 $\pm 229,62$ tiges/ha) et PAur8 (555,56 $\pm 229,62$ tiges/ha). La cinquième et dernière catégorie renferme uniquement PMan3 dont la valeur de densité moyenne est nulle.

Les surfaces terrières moyennes sont élevées dans PMan11 $\left(6,75 \pm 1,12 \mathrm{~m}^{2} / \mathrm{ha}\right)$ et PCra27 $\left(5,60 \pm 1,12 \mathrm{~m}^{2} / \mathrm{ha}\right)$, la plus petite valeur a été enregistrée dans PAur8 $\left(0,03 \pm 1,12 \mathrm{~m}^{2} / \mathrm{ha}\right)$ et a été notée, une valeur nulle dans PMan3. Les différences sont significatives $(P=7,893 ; F=0,000)$ et deux catégories de biotopes sont observées (Tableau V) : les biotopes aux valeurs de surfaces terrières moyennes élevées et ceux aux valeurs de surfaces de surface terrières moyennes faibles. Ce dernier groupe est composé de PAur8, PAur27, PMan3 et PMan8.

Tableau V : Valeurs moyennes des paramètres structuraux des biotopes étudiés

\begin{tabular}{cccc}
\cline { 2 - 4 } Biotopes & $\begin{array}{c}\text { Diamètre } \\
\text { moyen/individu }(\mathrm{cm})\end{array}$ & $\begin{array}{c}\text { Densité moyenne } \\
\text { (tiges/ha) }\end{array}$ & $\begin{array}{c}\text { Surface terrière } \\
\text { moyenne }\left(\mathrm{m}^{2} / \mathrm{ha} \text { ) }\right.\end{array}$ \\
\hline PAur8 & $3,01 \pm 0,56 \mathrm{~b}$ & $555,56 \pm 229,62 \mathrm{~cd}$ & $0,03 \pm 1,12 \mathrm{~b}$ \\
PAur27 & $3,57 \pm 0,56 \mathrm{~b}$ & $\mathbf{2 2 7 7 , 7 8} \pm \mathbf{2 2 9 , 6 2 a}$ & $0,36 \pm 1,12 \mathrm{~b}$ \\
PCra27 & $\mathbf{8 , 0 9} \pm \mathbf{0 , 5 6 a}$ & $1888,89 \pm 229,62 \mathrm{ab}$ & $\mathbf{5 , 6 0} \pm \mathbf{1 , 1 2 a}$ \\
PMan3 & $0,00 \pm 0,56 \mathrm{c}$ & $0,00 \pm 229,62 \mathrm{~d}$ & $0,00 \pm 1,12 \mathrm{~b}$ \\
PMan8 & $2,94 \pm 0,56 \mathrm{~b}$ & $1222,22 \pm 229,62 \mathrm{bc}$ & $0,10 \pm 1,12 \mathrm{~b}$ \\
PMan11 & $3,05 \pm 0,56 \mathrm{~b}$ & $\mathbf{2 6 6 6 , 6 7 \pm 2 2 9 , 6 2 a}$ & $\mathbf{6 , 7 5} \pm \mathbf{1 , 1 2 a}$ \\
\hline Paramètres du test & $P=21,946$ & $P=20,146$ & $P=7,893$ \\
& $F=0,0001$ & $F=0,0001$ & $F=0,000$ \\
\hline
\end{tabular}

*Pour chaque colonne, les valeurs moyennes affectées de la même lettre ne sont pas significativement différentes au seuil de $5 \%$ (test de Tukey) et les valeurs en gras sont les valeurs maximales.

\section{Discussion}

\section{Etude floristique}

\section{Richesse et diversité floristiques}

L'ensemble de la flore sous les peuplements d'Acacia, riche de 212 espèces et 158 genres est dominé par les familles des Fabaceae, Apocynaceae, Rubiaceae, Annonaceae, Euphorbiaceae. Les espèces les plus 
fréquentes sont Baphia nitida Lodd., Dichapetalum pallidum (Oliv.) Engl., Epinetrum cordifolium Mangenot \& Miege, Icacina mannii Oliv., Microdesmis keayana J.Léonard. Dans la même zone d'étude, Tiébré et al (2014) ont noté dans un inventaire, la dominance des Apocynaceae et Euphorbiaceae du côté de familles. Ils ont également signalé que Microdesmis keayana J.Léonard, Baphia nitida Lodd., Craterispermum caudatum Hutch., Dichapetalum dictyospermum Breteler et Hopea odorata Roxb étaient les espèces dominantes. Les richesses floristiques sont plus élevées dans les peuplements d'Acacia les plus âgés. Cela indique que la biodiversité s'améliore quantitativement en fonction de l'âge du peuplement. Cinq des six peuplements d'une part, et l'ensemble des relevés d'autre part sont dominés par les Fabaceae sensu lato. La dominance des Légumineuses a souvent été citée comme le trait botanique caractéristique des forêts ivoiriennes (Aubréville, 1959 ; Guillaumet et Adjanohoun, 1971). Toutefois, les forêts sempervirentes comme les forêts de la région Guinéo-Congolaise en général sont plutôt dominées par les Rubiaceae (Aké Assi, 1984 ; Sonké, 1998). Les indices de Shannon varient entre 2,33 et 3,02 avec des indices d'équitabilité élevés, ce qui témoigne d'une grande diversité biologique des différentes parcelles inventoriées. Cette analyse est différente de celle de Tiébré et al (2014) qui avaient observé une faible diversité de leurs parcelles d'étude dans la forêt de l’Anguédédou. En considérant chaque espèce d'Acacia (Acacia mangium Willd et Acacia auriculaeformis Benth notamment), on remarque la décroissance des indices de Shannon et de Piélou lorsqu'on part d'un stade jeune à un stade adulte. Cela traduit une reconstitution rapide dès les premiers âges des Acacias de la végétation ligneuse qui tend vers une augmentation du nombre d'espèces et une stabilité au fur et à mesure que ces peuplements d'Acacia vieillissent.

\section{Types morphologiques}

L'abondance des espèces arborescentes sous les différents peuplements d'Acacia traduit une excellente reconstitution de la végétation ligneuse sous l'effet de ces Légumineuses arborescentes. Dans l'ensemble des peuplements d'Acacia, l'étude a déterminé 49,06 \% d'espèces arborescentes, 29,72 \% d'espèces lianescentes et 21,23\% d'espèces herbacées. Ces proportions sont proches du postulat de Trochain (1980), selon lequel, en moyenne, les arbres et les arbrisseaux représentent $52 \%$ des espèces et les lianes ligneuses 32\%. Par ailleurs, la faible proportion d'espèces arborescentes enregistrées dans la parcelle d'Acacia mangium âgé de 11 ans s'explique par le fait que cette parcelle a subi quelques perturbations d'origines anthropiques avec notamment des pratiques culturales et des coupes de bois d'espèces d'Acacia mangium. Ces 
perturbations sont confirmées par le taux élevé d’espèces lianescentes dont la présence dans un milieu traduit son état de perturbation (Kouamé, 1998). La faible proportion d'espèces arborescentes enregistrée dans la parcelle d'Acacia mangium âgé de 3 ans s'explique par le fait que ce biotope est encore jeune et est dans une phase de début de mise en place de la biodiversité végétale dans ce milieu. Les proportions des différents types morphologiques de l'ensemble des relevés sont très proches de celles obtenues par Vroh et al., (2013) dans l'étude sur la Réserve botanique d'Agbaou.

\section{Etude structurale}

\section{Classes de diamètres}

L’ensemble des peuplements d'Acacia est dominé par des individus de petits diamètres. Sur les individus mesurés dans les placettes, 77,22 \% ont un diamètre compris entre $2,5 \mathrm{~cm}$ et $5 \mathrm{~cm}$. Cela montre que la végétation reconstituée est encore à un stade juvénile. Les seuls biotopes dans lesquels des individus de dbh supérieur à $5 \mathrm{~cm}$ ont été enregistrés sont les parcelles âgées de 27 ans d'Acacia auriculaeformis et Acacia crassicarpa qui sont d'ailleurs les peuplements les plus âgés. On note toutefois des différences significatives $(P=21,946 ; F=0,0001)$ entre les valeurs de diamètre moyen, avec notamment 5,10 $\pm 1,00 \mathrm{~cm}$ pour le peuplement de 27 ans de Acacia auriculaeformis et $11,56 \pm 1,00 \mathrm{~cm}$ pour le peuplement de 27 ans de Acacia crassicarpa. La forte présence des individus de petits diamètres est justifiée par le fait que la flore ligneuse naturellement régénérée a besoin d'un temps relativement long pour atteindre la maturité. Kassi et al. (2010) soulignent que la reconstitution des forêts qui est naturellement rapide dans les premières phases, aboutit à une résilience complète après au moins 40 ans.

\section{Densité et surface terrière}

Les densités moyennes de tiges par hectare dans chacun des peuplements sont élevées et varient considérablement (de 555,56 \$233,51 tiges/ha à 2666,67士233,51 tiges/ha). Les différences significatives observées $(P=20,146 ; F=0,0001)$ montrent que l'effet des Acacias sur la densité des espèces régénérées diffère selon le biotope. Ces valeurs moyennes obtenues sont largement supérieure à celles de Vroh et al. (2010). Ces auteurs avaient

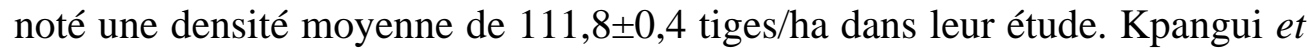

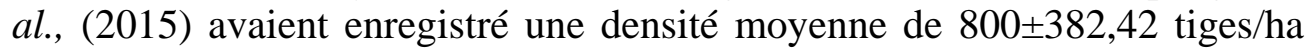
pour les individus de dbh supérieur à 2,5 cm dans des fragments de forêts de la Sous-préfecture de Kokumbo (Centre de la Côte d'Ivoire). Cette valeur est largement supérieure à celle du peuplement âgé de 8 ans d'Acacia auriculaeformis $(555,56 \pm 233,51$ tiges/ha) et inférieure à celles des autres biotopes, hormis le peuplement de 3 ans d'Acacia mangium où la densité 
moyenne est nulle. Les valeurs moyennes de surface terrière que nous y avons trouvé pour les dbh supérieurs à $2,5 \mathrm{~cm}$ sont comprises entre $0,92 \pm 40,35 \mathrm{~m}^{2} /$ ha et $13,08 \pm 40,35 \mathrm{~m}^{2} / \mathrm{h}$. Ce paramètre est très élevé pour cette classe de diamètre dans la parcelle d'Acacia mangium âgé de 11 ans $\left(242,91 \pm 40,35 \mathrm{~m}^{2} / \mathrm{ha}\right)$ et le peuplement de 27 ans d'Acacia crassicarpa

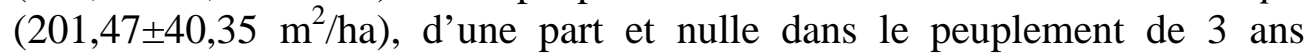
d'Acacia mangium. L’âge du peuplement a une influence positive sur l'occupation du sol par la biodiversité ligneuse; cela est prouvé par l'augmentation de la surface terrière en fonction de l'âge du biotope.

\section{Conclusion}

Cette étude nous a permis d'évaluer la diversité floristique et la structure de la flore sous des peuplements d'Acacia dans la station de recherche forestière d'Anguédédou, en zone de forêt dense humide sempervirente. Aussi, une reconstitution naturelle de la biodiversité ligneuse a été observée. Nous avons également enregistré une augmentation de l'occupation du sol sous ces peuplements par la flore ligneuse en fonction de l'âge des peuplements. Les indices de Shannon et d'équitabilité sont élevés avec des valeurs comprises respectivement entre 2,66 bits et 3.02 bits d'une part, et entre 0,95 et 0,96 d'autre part. Les types morphologiques les plus importants sont les arborescents et les paramètres structuraux (densité, diamètre moyen et surface terrière) sont élevés selon de l'âge du peuplement d'Acacia. La diversité floristique et la structure de la flore sous les peuplements d'Acacias constituent des valeurs écologiques et de résilience non négligeables. Elles sont des justificatifs suffisants pour une perspective de mise en valeur d'une utilisation non destructive de la forêt et de reforestation à l'échelle nationale. Ce travail recèle des résultats importants à prendre en compte dans la régénération de la biodiversité en général, de la flore ligneuse en particulier ainsi que pour l'amélioration et l'aménagement des forêts. Il s'agit d'apporter une méthode de travail et une stratégie pour favoriser le succès d'un projet de reforestation. Les préconisations du travail doivent être réalistes, notamment par la maîtrise de la régénération de la flore ligneuse sous des Légumineuses qui ont un impact positif sur la reconstitution de la végétation ligneuse.

\section{References:}

1. Adou K., (1986). Caractéristiques de la station CTFT de l'Anguédédou (1ère partie). Rapport technique CTFT, 7 pp.

2. Aké Assi L., (1984). Flore de la Côte d'Ivoire : étude descriptive et biogéographique, avec quelques notes ethnobotaniques. Thèse Doctorat, Université Nationale d'Abidjan (Côte d'Ivoire), 1206 pp. 
3. Aké-Assi L., (2001-2002). Flore de la Côte d'Ivoire : catalogue systématique, biogéographique et écologie. Boissiera. 57 : 396 pp. ; 58 : $401 \mathrm{pp}$.

4. Allou K., Aké S., Ahoussou N., Ballo K. et Diabaté S., (2001). Effet de la jachère sur l'expression de la fusariose vasculaire du palmier à huile (Elaeis guineensis Jacq.). Agronomie Africaine. 13 (1): 21-33.

5. APG III, (2009). An update of the Angiosperm Phylogeny Group classification for the orders and families of flowering plants. Bot. J. Linn. Soc. 161 (2): 105-121.

6. Aubréville A., (1959). Flore Forestière de la Côte d'Ivoire (Tomes 13). C.T.T. Nogent sur-Marne, Paris : Tome 1 : 372 pp. ; Tome 2 : 343 pp. ; Tome 3 : 335 pp.

7. Bertrand A., (1983). La déforestation en zone de forêt en Côte d'Ivoire. Bois et forêts des tropiques. 202 : 3-17.

8. Djaha B.A.O., N’Guessan A.K., Djaha J.B.A. et Traoré D., (2009). Impact des jachères à Légumineuses arborescentes sur la flore adventice au Sud de la Côte d'Ivoire. Agronomie Africaine. 21 (2) : 143-154.

9. Dupuy B. et N'Guessan K.A., (1991). Utilisation des acacias pour régénérer les anciennes cocoteraies. Bois et Forêts des Tropiques. $230: 15-29$.

10. Eldin M., (1971). Le climat de la Côte d'Ivoire. In Le Milieu Naturel de la Côte d'Tvoire, Avenard JM, Eldin E, Girard G, Sircoulon J, Touchebeuf P, Guillaumet JL, Adjanohoun E, Perraud A (eds). Mémoires de l’ORSTOM: 73-108.

11. Gibbs J.P., (1998). Distribution of woodland amphibians along a forest fragmentation gradient. Landscape Ecology, Vol. 13: 263-268.

12. Guillaumet J-L et Adjanohoun E., (1971). La végétation de la Côte d'Ivoire. In Le Milieu Naturel de la Côte d'Tvoire, Avenard JM, Eldin E, Girard G, Sircoulon J, Touchebeuf P, Guillaumet JL, Adjanohoun E, Perraud A (eds). Mémoires de l’ORSTOM : 157-263.

13. Havyarimana F., (2015). La contribution de l'instabilité sociopolitique dans l'anthropisation des paysages au Burundi : dynamique spatiale et biodiversité. Thèse de Doctorat en Sciences Agronomiques et Ingénierie Biologique, Université libre de Bruxelles (Belgique) : 184 pp.

14. Kassi N.J., Aké-Assi E. et Tiébré M.S., (2010). Biodiversité végétale et vitesse de la régénération de la forêt classée de Sanaimbo (Côte d’Ivoire). Sciences et Nature. 7 (2) :195-206.

15. Kouamé N'. F., (1998). Influence de l'exploitation forestière sur la végétation et la flore de la Forêt Classée du Haut-Sassandra (Centre- 
Ouest de la Côte d'Ivoire). Thèse de Doctorat 3e Cycle, Université de Cocody-Abidjan (Côte d'Ivoire) : 216 pp.

16. Kpangui K.B., Vroh B.T.A., Goné B.Z.B. et Adou Y.C.Y., (2015). Diversité floristique et structurale des cacaoyères du « v baoulé » : cas de la Sous-préfecture de Kokumbo (Centre, Côte d'Ivoire). European Scientific Journal. 11 (36) : 40-60.

17. N’Da D.H., Adou Y.C.Y., N’Guessan K.E., Koné M. et Sagné Y.C., (2008). Analyse de la diversité floristique du parc national de la Marahoué, Centre-Ouest de la Côte d'Ivoire. Afrique Science. 4 (3) : 552-579.

18. N’Goran A., (2005). Amélioration de la fertilité chimique des sables quaternaires en Côte d'Ivoire dans l'association cocotier/Acacias sp. Thèse de Doctorat, Université de GAND, (Belgique) : 193 pp.

19. N’Guessan K.A., Dupuy B., Assa A. et N'Goran A., (2006). Légumineuses arborescentes pour la gestion durable des terroirs agricoles en Basse Côte d’Ivoire. Agronomie Africaine. 18 (3) : 267283.

20. N’Guessan K.A., (1991). Contribution à l'étude de méthodes de régénération des jachères en basse Côte d'Ivoire : Le cas de l'Acacia mangium. Thèse de Docteur-Ingénieur, Université Nationale d'Abidjan (Côte d'Ivoire) : 103 pp.

21. N’Guessan K.A., (2006). Les légumineuses arborescentes, une alternative pour la régénération des jachères. Le cas de la zone forestière en Côte d'Ivoire. Thèse d'Etat ès Sciences Naturelles, Université de Cocody - Abidjan (Côte d’Ivoire) : 157 pp.

22. Quinn J.F. and Harrison S.P., (1988). Effects of habitat fragmentation and isolation on species richness: evidence from biogeographic patterns. Oecologia. 75: 132-140.

23. Sonké B., (1998). Études floristiques et structurales des forêts de la réserve de faune du Dja (Cameroun). Thèse de Doctorat, Université libre de Bruxelles (Belgique) : 267 pp.

24. Trochain, J.-L., (1980). Écologie végétale de la zone intertropicale non désertique. Publications Université Paul Sabatier, Toulouse : 458 pp.

25. Vroh B.T.A., Ouattara D., Kpangui K.B. et N’Guessan K.E., (2013). Diversité végétale et valeur pour la conservation de la réserve botanique d'Agbaou en création, Centre-ouest, Côte d'Ivoire. Journal of Animal \& Plant Sciences. 20 (1): 3034-3047.

26. Vroh B.T.A., Adou Yao C.Y., Kouamé D., N’DA K. D. et N'Guessan K.E., (2010). Diversité floristique et structurale sur le site d'une réserve naturelle volontaire à Azaguié, Sud-Est de la Côte d'Ivoire. European Journal of Scientific Research. 45 (3) : 411-421. 
27. Zakra N., (1996). Bilan positif de l'association Cocotier/Acacias pour la restitution de l'Azote. Plantation Recherche Développement : 18 pp. 